\title{
A Content Sharing System Using Dynamic Fog Consisting of Peer-to-Peer Terminals and Its Simple Evaluation
}

\author{
${ }^{1}$ Takuya Itokazu and ${ }^{2}$ Shinji Sugawara ${ }^{+}$ \\ ${ }^{1,2}$ Chiba Institute of Technology, Japan
}

\begin{abstract}
This paper proposes an efficient content sharing system by forming a Dynamic Fog with multiple Edge terminals that make up a Peer-to-Peer network and logically constructing a Cloud-Fog-Edge hierarchical structure. In general, by placing a Fog server between Cloud and an Edge terminal, redundant traffic between Cloud and Edge nodes can be reduced, and the delay time required for providing contents or transmitting some kind of directions from Cloud can be shortened by shortcuts using Fog. In this research, it is novel that the role of a Fog server is cooperatively played by multiple Edge terminals, instead of a relatively high-performance dedicated Fog server. The proposed system is evaluated by simple computer simulations in order to show its potential effectiveness.
\end{abstract}

Keywords: Cloud-Fog-Edge hierarchy, content sharing, Dynamic Fog, Peer-to-Peer.

\section{Introduction}

With the spread of high-speed and high-quality communication networks in recent years like $5 \mathrm{G}$ and the performance improvement of terminal devices such as PCs, tablet computers, and smartphones, the environment where a large number of users access a wide variety of contents such as images, videos, sounds, haptics, texts, and so on, and the environment where they are searching their favorite contents over the network or providing them each other have already been established. In addition, the effective use of abundant network resources including contents has been achieved.

In previous research, we proposed a content sharing system based on a hybrid of Peer-to-Peer network and Multi-Cloud, which assumed the contribution of user terminals, and clarified its effectiveness [1], [2], [3]. A system that executes a service combining a Cloud and Edge terminals generally has a two-layer structure of a Cloud layer and an Edge layer. When Edge terminals constitute a Peer-to-Peer network as shown in Fig. 1, or when each Edge terminal is working as a high-performance client with partial function of a server, the Edge terminals are connected to the system via the access network.

On the other hand, in recent years, in consideration of the situation around the Edge terminals, their regional characteristics, and user group preferences, etc. in order to provide services that respond to the needs that have been finely differentiated, in order to improve their response speed, or in order to offload network traffic and computational load, etc., computer systems with a three-layer structure in which an intermediate layer subsystem called Fog is arranged between the Cloud layer and the Edge layer, have been introduced and applied in various fields [4]. Such a three-layer structure is also sufficiently effective for content sharing, because it is expected that the index information which is unique to each region will be collectively shared in the Fog layer, and this causes that the contents will be exchanged quickly. However, if the Fog function is arranged as a dedicated server, the maintenance cost of the server will be caused in addition to the Cloud's. On

\footnotetext{
${ }^{+}$Corresponding author. Tel.: +81474780393.
}

E-mail address: shinji.sugawara@it-chiba.ac.jp 
top of that, it is necessary to keep this server even when there is no use opportunity, and it is difficult to make effective use of computational resources.

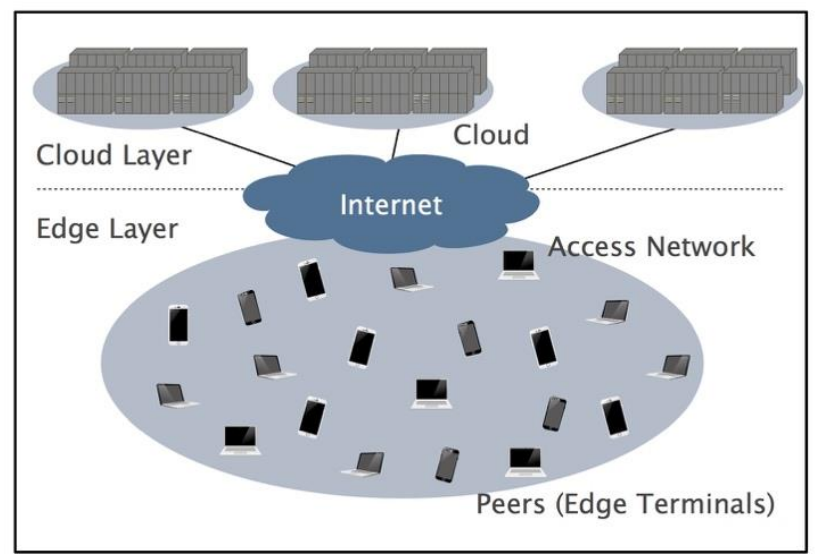

Fig. 1: Cloud-Edge Terminal: Two-Layer Structure.

We have already proposed to construct a content sharing system that combines the Peer-to-Peer-based system with a Cloud [1]. So, in this paper, the fundamental challenge is to dynamically compose a Fog function of intermediate layer by peers including mobile Edge terminals.

If it is possible to prepare a wide variety of mobile terminals, which compose a Peer-to-Peer network and share contents by coordinating with preliminarily prepared processes running on the Cloud, and if they in rotation and autonomously provide a necessary scale of virtual computing function of a Fog server according to each terminal's performance and situation, a very flexible and efficient content sharing system can be realized.

\section{Related Works}

Although there are not many similar proposals about the composition of a Fog function with dynamic nodes, the following two pieces of research have similar elements with ours.

In [5], a large number of terminals in the network are clustered into several groups, and each cluster is considered as one virtual machine. By allocating an appropriate load to each cluster, the authors aim to achieve a large-scale computation similar to the cloud.

In this research, they focus on dynamically switching and combining terminals in cluster generation, integrating them with a graph-theoretic approach, and mainly on appropriately distributing the computational load.

The purpose of our research is to share contents on the premise that the Edge terminals move, join, and leave the network autonomously, so there is a clear difference between this research and ours. On top of this, the system configuration is only terminals in this research, and there is no physical Cloud-Edge hierarchy like ours.

In [6], the system has a clear physical Cloud-Fog-Edge hierarchy, and the main idea is that Edge terminals can dynamically generate clusters according to the situation and be used for offloading of the Fog server.

It can be said that each Edge terminal is a part of the Fog server, but the Fog serer physically exists, and this research is very different from ours in the point that the Edge terminals are not combined according to the situation. The purpose of this research also seems to be efficient clustering with appropriate combinations of Edge terminals.

Unfortunately, the above two pieces of research have not been fully evaluated their effectiveness, and the objectives and main techniques are different from ours.

\section{Proposed Method}


As mentioned above, we have been conducting research on content sharing using Peer-to-Peer networks so far, and have proposed a method that aims to reduce storage capacity and network load, and improve content acquisition rate by adding a Cloud system.

And in recent years, in addition to simple systems consisting of Cloud and a group of peers which are a large number of user terminals, Fog computing and Edge computing aiming to shorten response time to users, to reduce network load, or to offload the server's processing running in the Cloud have attracted attention.

However, if Fog is used as an always-on server, it will be necessary to consider the costs of installation, management, and maintenance of the server, in addition to the Cloud's cost. In this case, it seems to be more difficult for us to evaluate the effectiveness of the system, such as what kind of cost we should assume as a system operating side, or who actually spends the cost.

Therefore, only two components of the system are used as before: The Cloud and a number of user terminals (Edge terminals) that make up the Peer-to-Peer network. In addition to performing Edge processing, peers dynamically configure a virtual Fog function in this paper, so a three-tier structure of Cloud-Fog-Edge can be logically established.

Although the resources equivalent to the server prepared by the system operating side are allocated in the required number of places near the terminals in normal Fog computing, in this research, this server function is virtually realized mainly by multiple user terminals that can be operated as high-performance Edge terminals, and they play a role of the Fog.

In such a system configuration, as shown in Fig. 2, the system provider can operate the system using only the resources allocated on the Cloud and the applications stored in each user Edge terminal. This makes the system configuration simple and can reduce the operating cost.

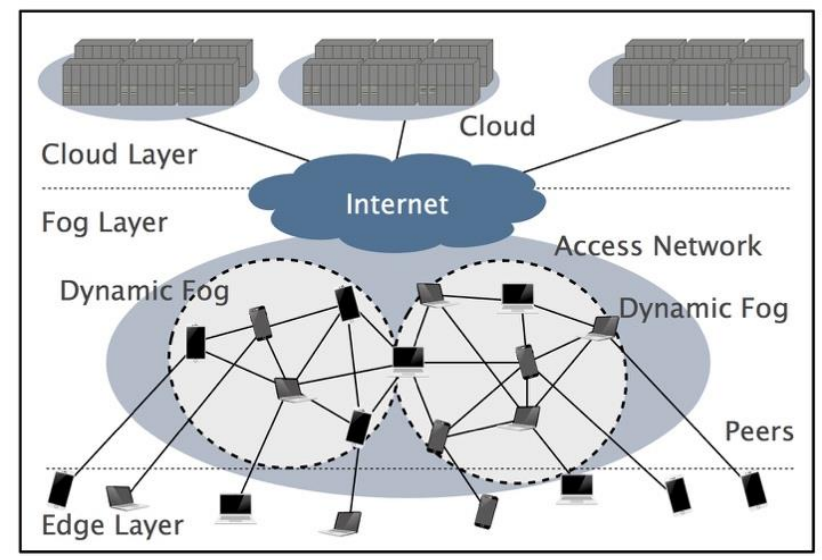

Fig. 2: System Configuration of Proposed System.

Although it is logically a Cloud-Fog-Edge three-layer system, it is physically a two-layer structure as shown in Fig. 1, and because a dynamic Fog can change its scale as necessary, there is no need to dedicate Fog resources during its unused periods of time and the system configuration can be efficient for both the system provider and the users.

Because dynamic Fog is logically configured, plural number of Fog functions are constructed at the same time for different purposes of content sharing such as with different shared genres, in different languages, or about regional differences, and each Edge terminal can join in the different plural number of Fog functions at the same time, too.

The core of the proposed system is the dynamic Fog part, and even if multiple Edge terminals move and repeatedly join and leave the Fog, the leader terminal, to be described below, cooperated with Cloud, tries to avoid the loss of the index information, which is needed for contents searching and allocation, and makes the system stable for the content sharing. The outline of the operation is described below. Note that we assume each Edge terminal on the access network, that can be a component of a Dynamic Fog in the initial state, is registered in the Cloud preliminarily.

1. The terminal to be the leader is determined by the Cloud according to the specification of each terminal. 
2. A dynamic Fog of appropriate scale is formed by the notification from the leader terminal to other terminals.

3. Periodic report including connection confirmation from each terminal to the leader using the access network is started.

From then, a content held by each terminal and the index information related to the content are shared and uploaded among terminals configuring Fog, when it is needed.

If there is a concern that the contents or index information may be lost due to the movement of the terminal, terminal's leaving the network, or the failure of the terminal, etc., the index information is appropriately transferred to another terminal, or copied and evacuated to the Cloud at the discretion of the leader.

General terminals connect to the terminals that configuring Fog, and always exchange its location information and possessing-contents information, and sometimes send content requests. In response to a content search request regardless of its sender, index information can be obtained by pure Peer-to-Peer system like behavior of the terminals configuring the Fog, and finally the requested content is sent from its owner to the requesting peer (terminal). If the requested content and its index information cannot be found in the Fog, the content is inquired to Cloud, and an attempt is made to obtain it from another Fog nearby or a Fog existing in another access network.

All the terminals move from one location to another, and join or leave the network at any time, and the terminals that make up the Fog jointly grasp them, and share index information and optimally relocate the contents.

\section{Evaluation}

In this paper, we evaluate the working effectiveness of the content sharing system described above, using a computer simulation under the simple scenarios and assumptions. In order to verify the effect of Dynamic Fog, we compare the results both when using Dynamic Fog and when not using it, under the environment where the Fog server is used. The simulator is made based on The One [7]. The main conditions set in the simulation are as follows.

- The nodes that make up the simulation are three types: Edge nodes, a Fog server, and Cloud.

- Up to 2,000, 3,000 and 4,000 Edge nodes (the average number of the nodes during the simulation is 1,250, 1,550 , and 1,850 , respectively) are randomly placed on $2 \mathrm{~km} \times 2 \mathrm{~km}$ plane in the initial state.

- Each node (considered Edge or user) is roughly equally divided into three categories based on its attributes (generation, etc.), and each node passes through 14 points which are randomly selected from 250 points on the plane, consisting of 125 points unique to each category and 125 points common to all categories.

- After that, each node moves to a specific point (assuming the starting or end point of high-speed transportation, such as a train station or an airport, etc.), and then disappears. Also, every 10 minutes, Edge nodes equivalent to $1 \%$ of total number of the nodes on the plane are newly added from this point.

- Each Edge node can exchange contents and queries by direct communication between nodes, and communication via base station of access network.

- Every 2 minutes, $20 \%$ of all Edge nodes are randomly selected, and each of these Edges makes a request for a single content to the Fog server via the base station of the access network. Then the location information of the requested content can be obtained from the Fog index information.

- There are 100 or 140 titles of content in total and these are all stored in the Cloud at any time, however the Fog and Edge nodes do not have any content in the initial state.

- Content capacity has a variety of $250 \mathrm{kB}, 500 \mathrm{kB}, 1 \mathrm{MB}, 3 \mathrm{MB}, 5 \mathrm{MB}$, and $10 \mathrm{MB}$, and their percentage of the total number of content titles is $25 \%, 25 \%, 15 \%, 15 \%, 10 \%$, and $10 \%$, respectively.

- The capacity that can hold the contents of each node is $100 \mathrm{MB}$, however it is $300 \mathrm{MB}$ only when the node serves as a part of Dynamic Fog. If it is necessary to hold a copy of a content exceeding this capacity, the one that had possessed at the earliest is deleted by FIFO.

- If an Edge node sends a content request to the Fog, and the Fog does not hold neither the content nor its index information, the Fog server gets the content from the Cloud, caches it in its own storage, and sends 
it to the requested Edge node. This Edge node is memorized by the Fog server as an Edge node having the content.

- The storage capacity of the Fog server is $600 \mathrm{MB}$ and if it is necessary to hold a copy of a content exceeding this capacity, delete some with LFU.

- The content requested by an Edge node is determined by the following procedure.

1. With a probability of $50 \%$, it is selected from all the titles of the contents.

2. Otherwise (for the case of the remaining 50\%), it is selected according to the popularity.

3. Each titles of the contents is classified into one of the three categories of top, middle, and low popularity, and each percentage of the total number of all titles is $20 \%, 30 \%$, and $50 \%$, respectively.

4. If the content title to be requested is selected according to the popularity, it is selected randomly from the categories of top, middle, and low popularity with the probability of $50 \%, 30 \%$, and $20 \%$, respectively.

- Some Edge nodes are converted to Dynamic Fog nodes so that the Edge nodes that make up Dynamic Fog occupy $10 \%$ of the entire Edge nodes once every 20 minutes. However, those Edge nodes should be decided taking care not to be close to each other. If there are no suitable Edge nodes, less than $10 \%$ will be acceptable.

- This simulation stated above is continued for 12 hours, and the number of communications (inquiries to Cloud, inquiries to the Fog server, communication through base stations between Edge nodes, and direct communication between Edge nodes), and the ratio of them are calculated. The calculated values are the average ones of simulation runs of 50 times.

Table 1: The main parameters of the simulation

\begin{tabular}{l|l}
\hline Parameters & Values \\
\hline \hline Maximum number of Edge nodes & $2,000,3,000,4,000$ \\
\hline Average number of Edge nodes & $1,250,1,550,1,850$ \\
\hline Number of content titles & 100,140 \\
\hline $\begin{array}{l}\text { Storage capacity of Edge node } \\
\text { (normal state) }\end{array}$ & $100[\mathrm{MB}]$ \\
\hline $\begin{array}{l}\text { Storage capacity of Edge node } \\
\text { (when serving as a part of Dynamic Fog) }\end{array}$ & $300[\mathrm{MB}]$ \\
\hline Simulation area & $2[\mathrm{~km}] \times 2[\mathrm{~km}]$ \\
\hline Capacity of a content title & $250[\mathrm{kB}], 500[\mathrm{kB}], 1[\mathrm{MB}\}, 3[\mathrm{MB}], 10[\mathrm{MB}]$ \\
\hline Storage capacity of Fog server & $600[\mathrm{MB}]$ \\
\hline Simulation period & $12[\mathrm{hours}]$ \\
\hline Number of simulation-runs & 50 \\
\hline
\end{tabular}

The main parameters included in the explanation of the simulation stated above is illustrated in Table 1. The result of the simulation is shown in Figs 3 through 7.

Fig. 3 and 4 show the ratio among communications of an Edge node to Cloud via a base station, to Fog server via a base station, to another Edge node via a base station, and to another Edge node directly, for content sharing by the procedure explained above, without using Dynamic Fog.

The difference between Fig. 3 and 4 is only the total number of sharing contents titles. The former shows in the case of 100 titles, and the latter shows in that of 140 titles. As the number of sharing contents titles increases, the ratio of content sharing between Edge nodes decreases, and that between an Edge node and Cloud or the Fog server increases. This is a reasonable result, because each Edge node has a small storage capacity and there is a limit to the number of copies of contents titles that can be stored, so if the total number of contents titles increases, content sharing should rely on Cloud or the Fog server.

Turning to Fig. 5 and 6 based on the result above, when Dynamic Fog is used together with the Fog server, it can be seen that the communication between Edge nodes increases rapidly whether via a base station or not. 
This indicates that Dynamic Fog has been able to almost replace the content sharing that relied on the Fog server, and it is considered that Dynamic Fog works effectively under the conditions given this time.

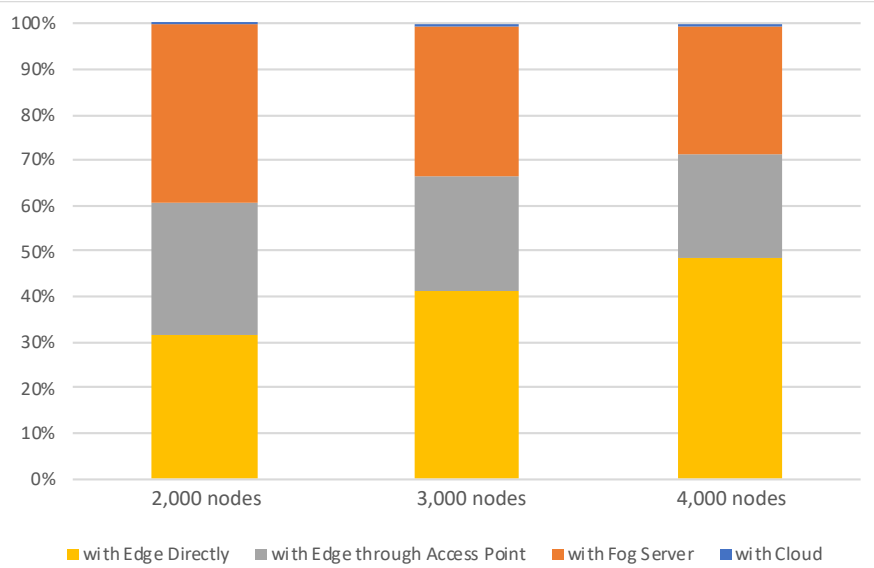

Fig. 3: Ratio of Communications (without Dynamic Fog, the number of contents titles is 100).

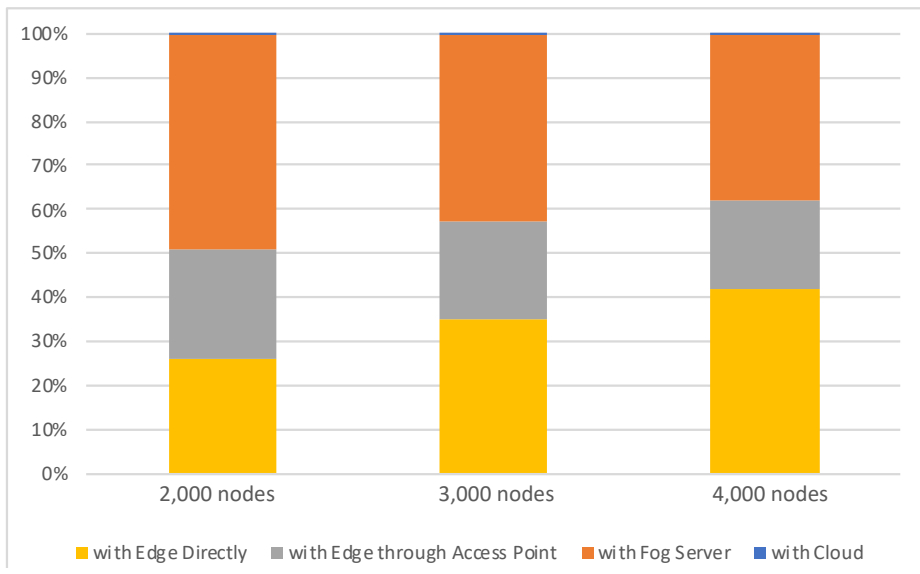

Fig. 4: Ratio of Communications (without Dynamic Fog, the number of contents titles is 140).

The difference between results of Fig. 5 and 6 is the total number of contents titles to be shared, so if this increases from 100 to 140, the usage ratio of Cloud and the Fog server increases just the same as the results when only the Fog server is used, shown in Figs. 3 and 4.

From Figs. 3 through 6, it can be seen that as the number of Edge nodes increases, the ratios of communications between Edge nodes without using a base station tend to increase. This is because, from the view point of each Edge node, the probability that there exist many other Edge nodes nearby that can cooperatively share contents is getting higher, and the content exchange which does not have to rely on the distant Edge nodes, the Fog server, nor Cloud to be connected via a base station is increasing.

Figure 7 shows the total number of communications in the case of using Dynamic Fog. As the number of Edge nodes increases, the number of communications increases, which is also a reasonable result.

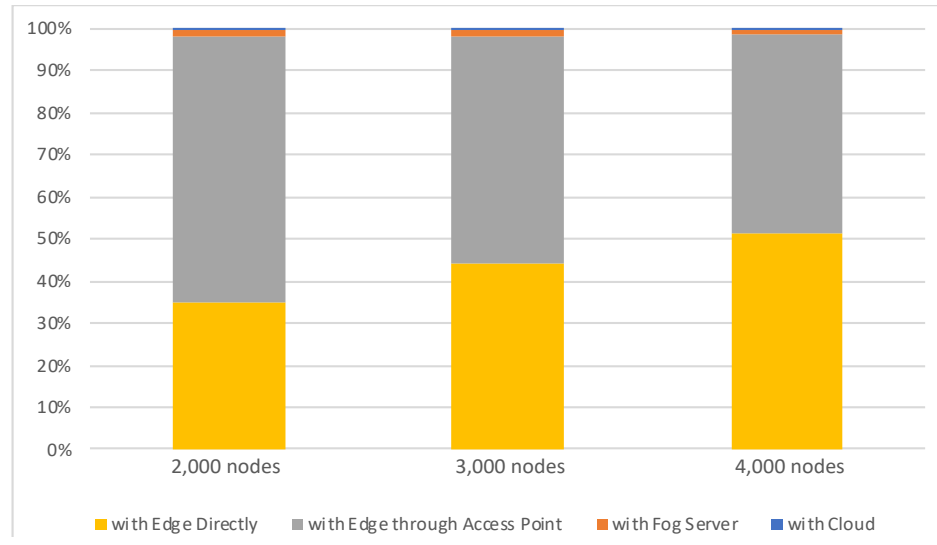

Fig. 5: Ratio of Communications (with Dynamic Fog, the number of contents titles is 100). 


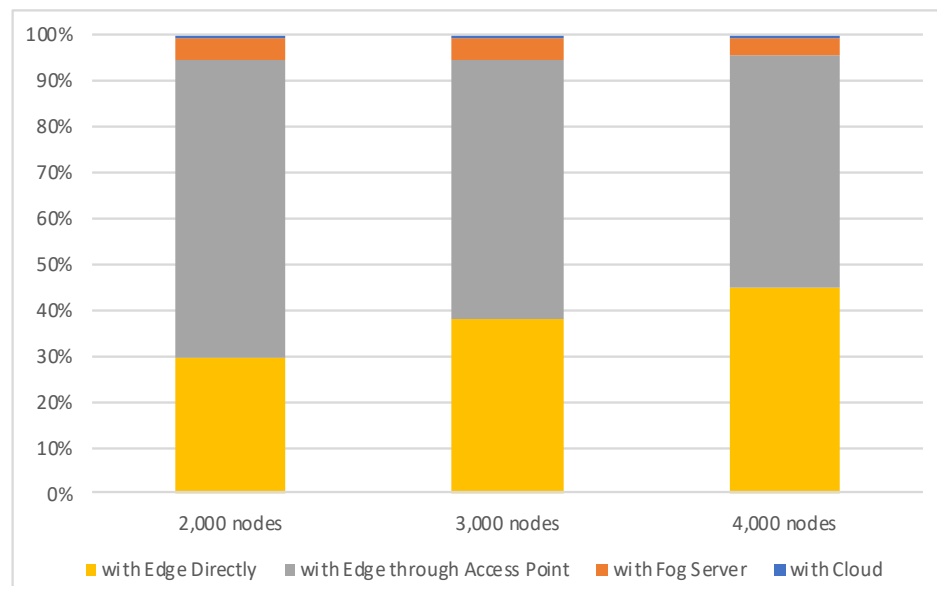

Fig. 6: Ratio of Communications (with Dynamic Fog, the number of contents titles is 140).

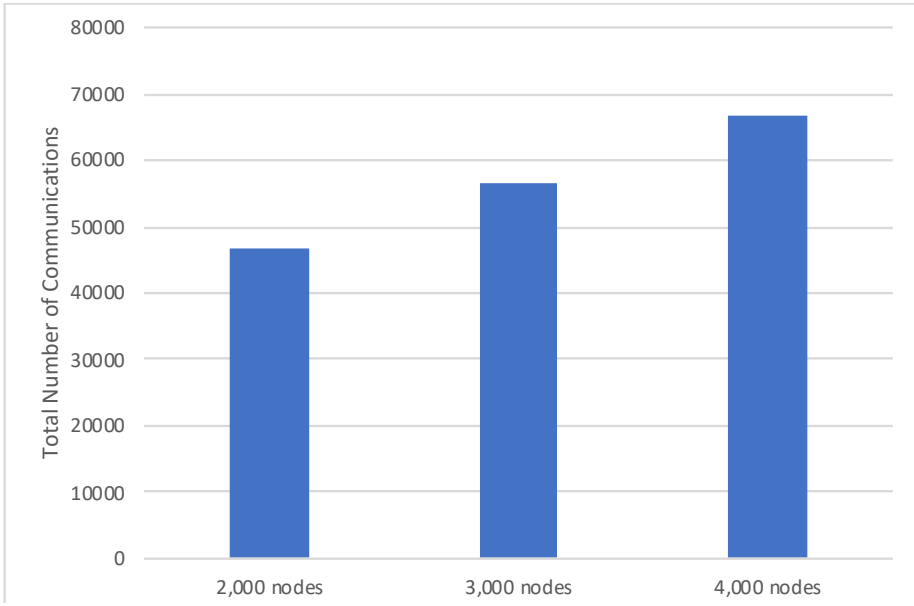

Fig. 7: Total Number of Communications.

From the above, it is clarified that the use of Dynamic Fog, at least under the condition set this time, could potentially replace the conventional Fog server function.

\section{Conclusion}

In this paper, we proposed an efficient content sharing system, which makes Dynamic Fog by the plural number of Edge nodes forming a Peer-to-Peer network. In Dynamic Fog, normal Edge nodes cooperate each other and replace the function of the conventional Fog server. Therefore, the content sharing system as a whole does not need to prepare dedicated resources for the Fog function, and flexible operation can be expected.

In order to confirm the effectiveness of the proposed method, we investigated by computer simulation how Dynamic Fog can replace the function of Fog server under a simple scenario. As a result, comparing the environment where only the Fog server was used and that where both were co-existed, it was revealed that the communication for content sharing largely depending on the Fog server in the former environment, could be replaced by Dynamic Fog in the latter one. From the above, it is considered that the proposed method has a potential effectiveness at least in the environment set this time.

As a future work, it is necessary to define the behavior of Dynamic Fog in more detail and to build a system that can be expected to operate more efficiently. It is also an important issue to evaluate the proposed method more accurately under the condition that is more realistic than that we set this time.

\section{References}

[1] M. Tomimori, S. Sugawara, "Content Sharing Method Using Expected Acquisition Rate in Hybrid Peer-to-Peer Networks with Cloud Storages," Int. J. Space-Based and Situated Computing, Vol. 7, No. 4, pp. 187-196, Feb. 2018.

[2] T. Murakami, S. Sugawara, "An Upload and Download Time Shortening Method for Multi-Cloud Content Sharing 
Systems," in Proc. 2019 IEEE International Conference on Consumer Electronics-Taiwan (ICCE-TW 2019), May 2019.

[3] T. Murakami, S. Sugawara, "Multi-cloud System for Content Sharing Using RAID-Like Fragmentation," Proc. The 22nd International Conference on Network-Based Information Systems (NBiS-2019), Advances in Intelligent Systems and Computing, 1036, pp. 49-60, Springer, Sep. 2019.

[4] S. Sugawara, "Survey of Cloud-based Content Sharing Research: Taxonomy of System Models and Case Examples," IEICE Trans. Commun., vol. E100-B, no. 04, pp. 484-499, April 2017.

[5] N. Song et al., "Fog Computing Dynamic Load Balancing Mechanism Based on Graph Repartitioning," China Communications, Vol. 13, Issue 7, pp. 156-164, Mar. 2016.

[6] Y. Li et al., "Dynamic Mobile Cloudlet Clustering for Fog Computing," Proc. International Conference on Electronics, Information and Communication (ICEIC 2018), Jan. 2018.

[7] The One, the opportunistic network environment Simulator: https://akeranen.github.io/the-one/ 